\title{
$\mathrm{D}$ 유형 성격이 관상동맥질환의 우울, 불안과 건강 관련 삶의 질에 미치는 영향: 체계적 문헌고찰 및 메타분석
}

배선형 $^{1 \odot}$. 박진희 ${ }^{2 \odot}$

아주대학교 간호대학 - 간호과학연구소 부교수 ${ }^{1}$, 아주대학교 간호대학 - 간호과학연구소 교수 ${ }^{2}$

\section{Impact of Type D Personality on Depression, Anxiety, and Health-related Quality of Life among Coronary Artery Disease Patients: A Systematic Review and Meta-analysis}

\author{
Bae, Sun Hyoung ${ }^{1} \cdot$ Park, Jin-Hee \\ ${ }^{1}$ Associate Professor, College of Nursing · Research Institute of Nursing Science, Ajou University, Suwon, Korea \\ ${ }^{2}$ Professor, College of Nursing · Research Institute of Nursing Science, Ajou University, Suwon, Korea
}

\begin{abstract}
Purpose: The purpose of this study was to investigate the impact of Type $D$ personality among patients with Coronary Artery Disease (CAD). Methods: Seven databases were searched to conduct a systematic review and meta-analysis. Two reviewers independently searched, selected, extracted, and assessed the studies. The quality of studies was evaluated using the JBI Critical Appraisal Checklist. To estimate the effect size, meta-analysis of the studies was performed using the Comprehensive Meta-Analysis 3.0 program. Results: Of 1,128 publications identified, 31 studies that met the inclusion criteria were used to estimate the effect size of Type D personality. Effect size (Standardized Mean Difference [SMD]) was used in the analyses. Patients with CAD who had Type $D$ personality had higher levels of depression ( $\mathrm{SMD}=0.92 ; 95 \%$ Confidecne Interval $(\mathrm{Cl})=0.74 \sim 1.10)$ and anxiety $(\mathrm{SMD}=1.19 ; 95 \% \mathrm{Cl}=0.81 \sim 1.57)$, but lower levels of physical and mental health-related quality of life $(\mathrm{SMD}=-0.56 ; 95 \% \mathrm{Cl}=-0.75 \sim-0.38 ; \mathrm{SMD}=-0.91$; 95\% $\mathrm{Cl}=-1.10 \sim-0.73)$. Publication bias was not detected. Conclusion: Type D personality was associated with increased depression and anxiety and impaired health-related quality of life in patients with CAD. Personality and psychosocial risk screening in patients with CAD should be conducted in the clinical setting.
\end{abstract}

Key Words: Type D personality; Coronary artery disease; Depression; Anxiety; Quality of life

\section{서 론}

\section{1. 연구의 필요성}

고령인구의 급격한 증가와 생활양식의 변화로 인해 발생률 이 높아지는 관상동맥질환은 국내 사망순위에서도 지속적으 로 높은 순위를 차지하고 있다. 협심증과 심근경색으로 대표되
는 관상동맥질환의 위험요인에는 흡연, 고혈압, 운동부족 등의 고전적인 요인들과 우울, 불안, 분노, 적대감, 성격유형 등의 심 리적 요인이 있는데 이 중 중요하게 고려되는 하나가 D 유형 성 격이다[1-4]. 이는 시간과 장소에상관없이 분노, 슬픔, 공포, 불 안정과 같은 부정적인 감정을 경험하는 경향인 부정적 정서 (Negative Affectivity, NA)와 사회생활 중 타인에게 거부당 할 잠재적 두려움을 피하기 위해 감정과 행동을 억제하는 경향

\section{주요어: D유형 성격, 관상동맥질환, 우울, 불안, 삶의 질}

Corresponding author: Park, Jin-Hee https://orcid.org/0000-0002-0069-7819

College of Nursing, Ajou University, 164 Worldcup-ro, Yeongtong-gu, Suwon 16499, Korea.

Tel: +82-31-219-7019, Fax: +82-31-219-7020, E-mail: jhee@ajou.ac.kr

Received: Mar 7, 2019 / Revised: Apr 15, 2019 / Accepted: May 8, 2019

This is an open access article distributed under the terms of the Creative Commons Attribution Non-Commercial License (http://creativecommons.org/licenses/ by-nc/3.0), which permits unrestricted non-commercial use, distribution, and reproduction in any medium, provided the original work is properly cited. 
인 사회적 제한(Social Inhibition, SI)의 기질을 동시에 가지는 것을 말한다[1].

$\mathrm{D}$ 유형 성격을 가진 사람들은 부정적 정서와 사회적 제한이 강하기 때문에 불안이나 걱정, 불쾌감을 경험하기 쉽고 자신을 부정적으로 바라보는 경향이 있으며, 다른 사람과 있을 때 더 긴장하고 불안정한 양상을 가지기 쉬운 것으로 알려져 있다 [1]. 이러한 $\mathrm{D}$ 유형 성격의 특성으로 인해 $\mathrm{D}$ 유형 성격을 가진 관상동맥질환자는 우울[5-7,A27]이나 불안[7,A27,A28]과 같 은 정서적 건강문제를 더 많이 경험한다. 우울과 불안은 심혈관 질환자에게 흔히 보고되는 정서적 건강문제로 $[8,9]$ 심혈관 질 환으로 인한 사망률이나 이환율, 그리고 재발위험을 높이는 주 요 요인으로 고려되고 있다[8,10,11]. 그뿐만 아니라 $\mathrm{D}$ 유형 성 격을 가진 사람은 흥통, 호흡곤란과 같은 심혈관 증상을 자주 느끼거나 증상경험의 중증도가 높음에도 불구하고[12] 의료진 에게 본인의 증상을 보고하지 않는 경향이 높고[13], 건강한 식 이습관, 운동, 정기 검진 등의 건강행위의 수행률이 낮으며[14] 적절한 의료서비스를 찾아 이용하려는 경향이 적어서 건강상 태와 건강 관련 삶의 질이 나쁘다고 알려져 있다[3,7,12-14].

최근 치료요법의 발달과 더불어 관상동맥질환자들의 생존 기간이 길어지면서 이들의 삶의 질을 향상시키기 위한 관심이 증가하는 추세이다[15]. 건강 관련 삶의 질은 치료요법에 대한 환자의 만족도를 평가하는 중요한 환자보고형 결과로[15], 관 상동맥질환과 같은 만성질환자의 예후와 관련이 높은 것[16] 으로 알려짐에 그 중요성이 강조되고 있다. 그러나 최근 장기간 의 추적관찰연구에 따르면 관상동맥질환자는 건강인에 비해 삶의 질이 낮은 것으로 보고된다[15]. 그러므로 관상동맥질환 자의 건강 관련 삶의 질을 향상시키고 우울과 불안을 감소시키 며 건강한 생활습관을 영위하여 이차건강문제를 예방하기 위 해서는 건강 관련 삶의 질과 증상관리에 영향을 미치는 주요 요 인인 D유형 성격에 대한 관심과 연구가 필요하다[3,A16,A19].

이에 국외에서는 $\mathrm{D}$ 유형 성격에 대한 다양한 연구가 활발히진 행되고 있는 편으로, 삽입형제세동기(implanted a cardioverter defibrillator) 환자를 대상으로 $\mathrm{D}$ 유형 성격이 우울, 불안과 건 강상태에 미치는 영향을 규명하거나[7], 말초혈관질환자나 심 부전 환자를 포함한 심혈관질환자를 대상으로 $\mathrm{D}$ 유형 성격이 신체적 건강상태와 주요 심장 사건(Major Adverse Cardiac Events, MACE)의 발생위험을 분석하기도 하였으며 $[16,17]$ 최근에는 일반인[18]과 심질환자를 제외한 만성질환을 가진 대상자를 중점으로[19] D유형 성격이 건강상태에 미치는 영향 에 대한 체계적 문헌고찰 연구가 보고되기도 하였다. 이렇듯 D 유형 성격이 소개된 이래 주로 관상동맥질환을 포함한 심질환
환자들을 중심으로 연구가 이루어졌다. 그러나 관상동맥질환 자를 대상으로 수행된 연구들[1-3]은 D유형 성격이 관상동맥 질환자의 심인성 혹은 비심인성 사망비나 $\mathrm{MACE}$ 의 발생위험 의 효과 크기만을 제시하고 있는데 국한되어 있어서, $\mathrm{D}$ 유형 성 격이 우울, 불안과 건강 관련 삶의 질과 같은 환자보고형 결과 에 미치는 영향을 종합하여 분석한 연구는 미흡한 실정이다. 더욱이 국내에서도 지금까지 심질환자를 대상으로 한 $\mathrm{D}$ 유형 성격에 대한 연구가 일부 진행되기는 하였지만 $\mathrm{D}$ 유형 성격 관 련 논문의 분석[9], $\mathrm{D}$ 유형 성격 측정도구의 타당도와 신뢰도 검증 [20,A14]을 제외하고는 D유형 성격에 따른 관상동맥질환 자의 건강 관련 삶의 질과 건강상태, 건강행위[A21,A25]의 차 이를 조사한 연구들에 국한되어 있다. 따라서 본 연구에서 체 계적 문헌고찰을 적용하여 $\mathrm{D}$ 유형 성격이 관상동맥질환자의 우울, 불안과 건강 관련 삶의 질에 미치는 영향을 규명해봄으로 써 앞으로 $\mathrm{D}$ 유형 성격이 가지는 중요성에 대한 관심을 유도하 고 $\mathrm{D}$ 유형 성격 관련 연구의 활성화 및 간호실무에서의 활용을 기여하고자 한다.

\section{2. 연구목적}

본 연구의 목적은 관상동맥질환자에서 $\mathrm{D}$ 유형 성격이 미치 는 영향을 확인하고 추후 이들 환자의 삶의 질 향상을 위한 중 재개발의 기초자료를 제공하는 것으로 구체적인 목적은 다음 과 같다. 첫째, 관상동맥질환자의 $\mathrm{D}$ 유형 성격에 따른 우울, 불 안과 건강 관련 삶의 질을 조사한 연구를 체계적으로 고찰한다. 둘째, 체계적으로 고찰한 선행연구의 일반적 특성을 확인한다. 셋째, 선행연구를 메타분석하여 $\mathrm{D}$ 유형 성격이 우울, 불안과 건 강 관련 삶의 질에 미치는 영향에 대한 효과크기를 파악한다.

\section{연구방법}

\section{1. 연구설계}

본 연구는 관상동맥질환자에서 $\mathrm{D}$ 유형 성격이 우울, 불안과 건강 관련 삶의 질에 미치는 영향을 통합하고 분석하기 위한 체 계적 문헌고찰 및 메타분석 연구이다.

\section{2. 연구선정기준}

연구선정을 위하여 관상동맥질환자의 $\mathrm{D}$ 유형 성격이 우울, 불안과 건강 관련 삶의 질에 미치는 영향을 파악하기 위한 고찰 
질문인 핵심질문(Participants, Intervention, Comparisons, Outcomes, Study Design, PICO-SD)을 선정한 뒤 선택, 배제 기준에 따라 국내·외 전자 데이터베이스 검색을 시행하였다.

\section{1) 선정기준}

자료검색의 선정기준의 경우 (1) 연구대상(participants)은 관상동맥질환의 의미하는 ST분절상승심근경색(ST-segment Elevation Myocardial Infarction, STEMI), 비ST분절상승심 근경색(Non-ST-segment Elevation Myocardial infarction, NSTEMI), 안정형, 불안정성, 이형성 협심증(unstable angina) 을 진단받은 성인이다. (2) 중재(intervention)는 Denollet [1] 이 개발한 Type D Personality Scale (DS-14, DS-16, DS-24) 를 이용하여 측정한 점수가 부정적 정서 하위영역 그리고 사 회적 제한 하위영역이 동시에 기준점(DS14의 경우 영역별 합산 10점) 이상으로 분류된 것으로 하였다. (3) 비교 대상 (comparisons)은 Denollet [1]이 개발한 Type D Personality Scale (DS-14, DS-16, DS-24)를 이용하여 측정한 점수가 부정 적 정서 하위영역 혹은 사회적 제한 하위영역 중 하나라도 기준 점(DS14의 경우 영역별 합산 10점 미만)인 경우로 분류된 것으 로 하였다. (4) 결과(outcomes)는 D유형 성격 여부에 따라 자 기 보고로 측정된 우울, 불안과 건강 관련 삶의 질의 분석가능 한 정량적 값을 선택하였다. (5) 연구유형(types of studies)은 횡단적, 종적 조사연구를 연구유형으로 선택하였다.

\section{2) 배제기준}

자료분석을 위해 (1) 관상동맥질환 외 다른 질환자를 포함 한 연구, (2) 독립변수로서 $\mathrm{D}$ 유형 성격을 측정하지 않았거나 $\mathrm{D}$ 유형 성격을 Denollet [1]이 개발한 Type D Personality Scale (DS-14, DS-16, DS-24)를 이용하여 측정하지 않았거나, 혹은 측정한 점수를 분류기준에 따라 $\mathrm{D}$ 유형 성격 여부로 분류 하지 않은 연구(예, $\mathrm{D}$ 유형 성격의 하위영역의 평균값을 제시한 경우), (3) 우울, 불안과 건강 관련 삶의 질 이외의 변수를 결과 변수(예, 사회적 지지, 직장복귀, 심장재활 프로그램의 참여 등)로 측정하였거나 통계치를 제시하지 않은 연구, (4) 실험연 구(순수, 유사, 원시 실험연구), 질적연구, 메타분석, 종설, 동물 및 임상시험연구는 배제하였다.

\section{3. 연구검색 및 선정}

본 연구는 코크란 연합(Cochrane collaboration)의 체계 적 문헌고찰 핸드북[21]과 Preferred Reporting Items for
Systematic Reviews and Meta-Analyses (PRISMA) 그룹이 제시한 체계적 문헌고찰 보고지침[22]에 따라 진행되었다.

\section{1) 자료 검색}

자료 검색은 $\mathrm{D}$ 유형 성격이라는 개념이 처음 등장한 1995 년 1월 1일부터[1] 2018년 12월 31일 이전까지 출간된 연구를 대상으로 하였다. 검색을 수행한 기간은 2019년 1월 10일부 터 2019년 1월 26일까지이다. 연구검색에 앞서 핵심질문 (PICO)을 바탕으로 각 데이터베이스별 키워드를 선정하고 의학주제표목(Medical Subject Headings, MeSH)와 생명 과학분야 용어색인(EMBASE Tree, EMTREE)을 이용한 검 색전략을 수립하였다. 국외 검색은 의학분야 전자데이터베 이스(OVID Medline \& PubMed), 유럽의학분야전자데이터 베이스(Excerpta Medica dataBASE, EMBASE), 간호보건분야 전자데이터베이스(Cumulative Index to Nursing and Allied Health Literature, CINAHL), PsycINFO/PsycARTICLES 를, 국내 검색은 대한의학학술지편집인협의회(KoreaMed), 학 술연구정보서비스(Research Information Sharing Service, RISS)를 이용하였다. 그리고 최종 선정된 연구의 참고문헌목 록을 검토하여 데이터베이스에서 검색되지 않은 연구는 수 기로 검색하였다. 검색식은 먼저 $\mathrm{MeSH}$ 용어와 제목과 초록 의 text word를 AND/OR 및 절단 검색을 적절히 적용하였 다. 검색을 위해 사용된 주요 핵심어는 대상자는 영어로는 ('myocardial ischemia' [Mesh] OR 'coronary artery disease' [Mesh] OR myocardi* infarction OR myocardi* ischemia OR coronary artery disease OR *stable angina OR variant angina OR acute coronary syndrome), 한국어로 는 (심근경색 $\mathrm{OR}$ 관상동맥질환 $\mathrm{OR}$ 관동맥질환 $\mathrm{OR}$ 심근허혈 $\mathrm{OR}$ 불안정형 협심증 $\mathrm{OR}$ 이형성 협심증 $\mathrm{OR}$ 변이형 협심증 $\mathrm{OR}$ 급성관상동맥증후군)이고, 중재는 영어로는 ('type D personality' [Mesh] OR type D personality OR distressed personality OR negative affect* OR social inhibit*), 한국어 로는 $(\mathrm{D}$ 유형 성격 $\mathrm{OR} \mathrm{D}$ 유형 인격 $\mathrm{OR}$ 디스트레스 성격 $\mathrm{OR}$ 부 정적 정서 $\mathrm{OR}$ 사회적 제한)이고, 결과는 영어로는 ('quality of life'[Mesh] OR 'anxiety' [Mesh] OR 'depression' [Mesh] OR quality of life OR anxi* OR depres*), 한국어로는 (삶의 질 OR 불안 OR 우울)을 이용하였으며, 영어 및 한국어로 발표 된 연구로 제한하였다.

\section{2) 자료수집과 선별}

2 명의 연구자가 독립적으로 자료수집과 선별의 전 과정을 
실시하였고, 의견이 일치하지 않으면 합의점을 찾을 때까지 기준에 따라 연구를 검토하였다. 데이터베이스를 통해 검색된 연구의 목록을 작성한 후 서지반출 프로그램인 엔드노트 (EndNote X9)을 이용하여 중복 연구를 제외하였다. 다음으로 제목과 초록을 검토하여 연구 선정기준에 부합된 연구인지를 확인하였으며, 선정기준에 부합하는지 판단하기 어려운 경우 본문을 참조하여 선정여부를 결정하였다. 모든 연구의 서지정 보를 동일하게 관리하였으며, 배제되는 연구에 대해서는 단계 별로 기록을 남겼다.

\section{3) 연구의 질 평가}

최종 선정된 연구의 질 평가는 관찰연구 보고의 질을 향상시 키고자 개발된 Joanna Briggs Institute (JBI) [23]의 질평가 도 구를 사용하였다. 최종 선정된 연구에 코호트 설계의 연구도 포함되었으나 본 연구의 목적은 $\mathrm{D}$ 유형 성격에 따른 결과변수 의 차이를 비교하는 것으로, 횡단적 조사연구를 위한 질평가 도구(Critical Appraisal Checklist for Analytical Cross Sectional Studies)를 이용하여 질평가를 실시하였다. 본 도구 는 '연구대상자 포함기준과 제외기준의 명확성', '표본 추출 장 소와 시간 및 대상자의 특성', '독립변수 측정', '대상자 선정에 대한 구체적인 기술' ' '혼동변수의 정의', '혼동변수의 통제', '결과변수의 측정', '통계 분석방법의 적절성'의 총 8 문항으 로 구성되어 있다. 각 문항은 예(Yes), 아니오(No), 불명확 (Unclear), 해당 사항 없음(Not applicable) 중 한 개에 응답하 도록 되어 있다. JBI 질평가 도구는 논문의 질을 평가하는 양적 기준을 제시하지 않으며 평가문항의 적절성을 검토하도록 하 고 있다. 이에 본 연구에서는 문헌의 질 평가를 두 명의 연구자 가 각각 독립적으로 수행하였으며, 일치되지 않는 항목은 합의 점을 찾을 때까지 해당 연구를 함께 검토하였다.

\section{4. 자료분석}

최종 선정된 연구는 관상동맥질환에서 $\mathrm{D}$ 유형 성격 연구의 정보를 수집하기 위해 연구자가 제작한 코딩표에 자료를 코딩 하였다. 코딩표에 입력된 자료는 저자, 출판년도, 출판국가, 연 구설계, 연구대상자의 진단명, 연구대상자 수, $\mathrm{D}$ 유형 성격 측 정도구, 독립변수 및 측정도구, 추후조사기간, 결과변수 및 측 정도구, 통계치(통계분석방법 및 보정변수)이며, 코딩표를 이 용하여 연구의 일반적 특성을 분석하였다.

메타분석은 연구 선정기준에 따라 분석에 필요한 통계치를 확인할 수 없는 연구들이나 결과변수의 하부척도 점수만을 제
시한 연구들을 제외한 31편을 대상으로 실시하였다. 결과변수 중 건강 관련 삶의 질은 신체적 안녕 및 정신적 안녕을 구분하 여 효과크기를 산출하였다. 그리고 한 연구에서 하나의 결과변 수를 2 개의 도구를 이용하여 측정한 경우에는 다른 연구에서 주로 사용되는 도구로 측정한 통계치를 분석에 포함하였다. 또 한 종적 조사연구에서 결과변수를 시점별로 측정한 경우에는 본 연구에서는 $\mathrm{D}$ 유형 성격과 동일한 시점에 측정한 점수만을 이용하였다.

$\mathrm{D}$ 유형 성격의 효과크기, 이질성 및 출판비뚤림에 대한 통계분 석은 Comprehensive Meta-Analysis ${ }^{\circledR} 3.0$ 프로그램(Biostat, Englewood, NJ, USA)을 이용하였다. 먼저 효과크기는 각 연 구의 결과값을 단일 단위로 표준화한 통계량인 표준화된 평균 차(Standardized Mean Difference, SMD)를 이용하여 계산 하였으며, 효과크기는 각 연구의 표본 특성, 비교 상태가 다양 하다는 점을 고려하여 변량효과모형(random- effects model) 을 적용하여 산출하였다. 먼저 연구의 효과크기의 이질성 (heterogeneity)을 평가하기 위하여 숲그림(forest plot)을 통 해 시각적으로 살펴보았다. 그리고 전체 관찰된 분산인 $\mathrm{Q}$ 통계 량을 산출하여 카이제곱검증을 하였으며, 이질성이 발견되면 전체 관찰된 분산에 대한 실제 분산인 연구간 분산의 비율을 나 타내는 Higgin의 $\mathrm{I}^{2}$ 값을 산출하여 $\mathrm{I}^{2}$ 가 $0.0 \%$ 일 때는 이질성이 없음으로, $50.0 \%$ 는 중간정도의 이질성, $75.0 \%$ 이상은 이질성 이 큰 것으로 판단하였다[21]. 그리고 각 연구가 보여주는 효 과크기의 이질성에 대한 추가적인 설명을 위해 연구 수준의 특성 즉, 조절변수의 속성에 따라 메타회귀분석을 활용하여 조절효과분석을 실시하였다. 한편 검색된 연구들의 출판비뚤 림은 깔때기 도표, Egger's linear regression asymmetry test 를 이용하여 검정하였으며, 검정결과에 대한 신뢰도는 안전계 수(Fail-safe N, Nfs)로 판단하였다. 안전계수는 종합적 치료 효과 크기가 유의하지 않은 것으로 판정되기 위해 숨겨진 연구 의 수를 산출한 것으로, 안전계수가 '충분히 크다'라고 판단할 수 있는 기준은 보편적으로 $5 n+10(n=$ 분석에 포함된 연구의 수) 이상이어야 한다.

\section{연구결과}

\section{1. 자료 선정}

데이터베이스별로 Medline (OVID와 PubMed)을 통해 223편, EMBASE을 통해 717편, CINAHL을 통해 55편, PsycINFO/PsycARTICLES을 통해 107편, KoreaMed을 통해 6 
편, RISS을 통해 16 편, 수기 검색을 통해 4 편인 총 1,128 편의 연 구가 검색되었다. 1,128 편 중 서지관리 프로그램을 이용하여 중복된 연구인 543 편을 제외하였고 585 편의 연구를 자료선정 및 제외기준에 따라 2 명의 연구자가 제목과 초록을 중심으로 검토하였다. 그 결과, 검색된 585 편 중 84 편을 1 차 선별한 뒤 포 함기준의 적합여부와 검색전략으로 사용한 검색어가 모두 포 함되었는지를 원문으로 확인하였다. 84편의 연구 가운데 연구 대상자의 선정기준에 부합하지 않은 연구 18 편, $\mathrm{D}$ 유형 성격을 측정하지 않은 연구 7편, 결과변수가 우울, 불안과 건강 관련 삶 의 질이 아니거나 혹은 분석에 적합한 통계치를 제시하지 않은 19편, 연구설계가 선정기준에 부합되지 않는 연구 1편 등 45편 을 제외한 후 39 편을 선별하였다. 39 편 중 연구대상자와 연구 설계가 동일하나 연구결과를 나누어 보고한 연구와 다년도간 연구를 진행하여 결과를 연차적으로 보고한 연구 15 편을 7 편 으로 연구결과를 병합하여 최종 31편을 체계적 문헌고찰 및 메 타분석을 위한 연구로 선정하였다(Figure 1).

\section{2. 분석에 포함된 연구의 질 평가}

분석에 포함된 31 편의 연구에 대한 질 평가 결과, 8 개의 평가 항목 중 '연구대상자 포함기준과 제외기준을 명확하게 제시'한 연구가 $96.8 \%$, '표본 추출 장소와 시간 및 대상자의 특성을 구 체적으로 제시'한 연구가 $100.0 \%$, '독립변수를 타당하고 신뢰 성 있게 측정'한 연구는 $100.0 \%$, '대상자 선정에 대해 구체적으 로 기술'한 연구는 $100.0 \%$, '혼동변수의 정의'한 연구는 $54.8 \%$, '혼동변수의 통제'한 연구는 $77.4 \%$, '결과변수를 타당하고 신 뢰성 있게 측정'한 연구는 $100.0 \%$, '적절한 방법으로 통계분석 을 실시'한 연구는 $100.0 \%$ 였다(Table 1).

\section{3. 분석에 포함된 연구의 일반적 특성}

분석에 포함된 총 31편 중 18편(58.1\%)의 연구가 2011년과 2015년 사이에 게재되었으며, 지역별로는 7편(22.6\%)의 연 구가 한국에서 실시되었으며 국외에서는 8 편 $(25.8 \%)$ 이 네

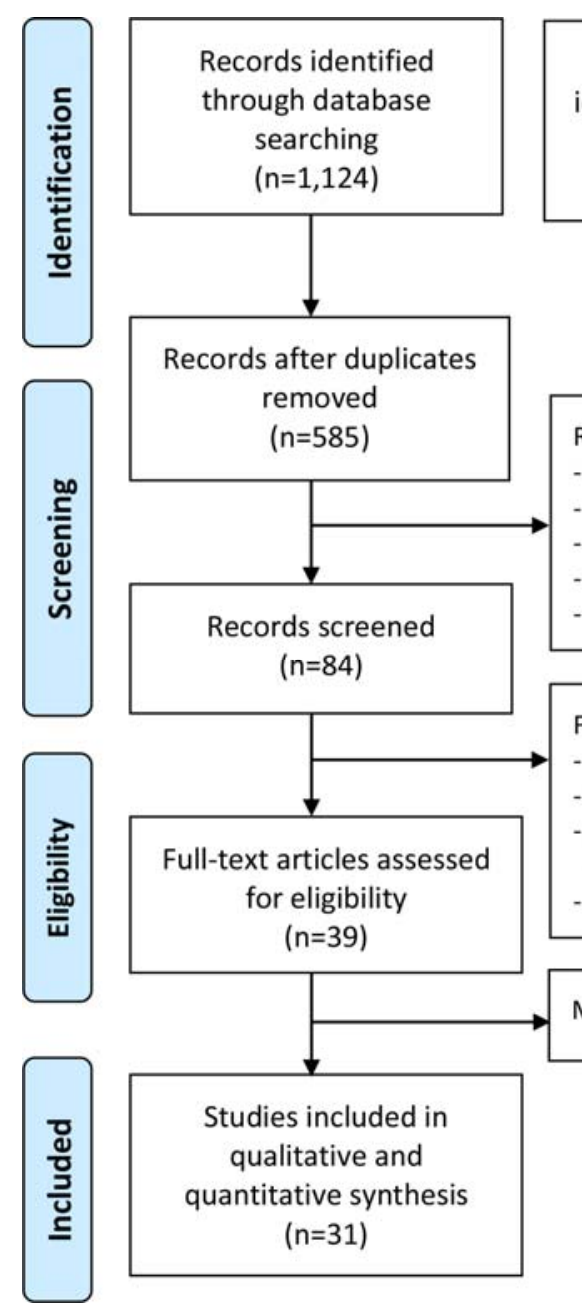

Additional records identified through other sources $(n=4)$

Records excluded by title \& abstract review $(n=501)$

- Not matched participants inclusion criteria $(n=117)$

- Not test the Type D personality $(n=149)$

- Not measured psychosocial outcomes $(n=184)$

- Not matched study design criteria $(n=13)$

- Not matched publication type $(n=38)$

Full-text articles excluded, with reasons $(n=45)$

- Not matched participants inclusion criteria $(n=18)$

- Not test the Type D personality $(n=7)$

- Not measured or reported statistics of psychosocial outcomes $(n=19)$

- Not matched study design criteria $(n=1)$

Merged 15 articles into 7 articles

Figure 1. Flow diagram for study selection. 
Table 1. Descriptive Summary of Included Studies

\begin{tabular}{|c|c|c|c|c|c|c|c|c|c|}
\hline Author & Country & Design & $\mathrm{n}$ & $\begin{array}{c}\text { Age } \\
\mathrm{M} \pm S \mathrm{SD}\end{array}$ & $\begin{array}{c}\text { Gender } \\
\mathrm{n}\end{array}$ & Diagnosis & $\begin{array}{l}\text { Type D } \\
\text { Scale, \% }\end{array}$ & $\begin{array}{l}\text { Outcome } \\
\text { scale }\end{array}$ & $\begin{array}{l}\text { Quality } \\
\text { score }\end{array}$ \\
\hline $\begin{array}{l}\text { Annagür et al. } \\
2016 \text { [A1] }\end{array}$ & Turkey & CS & 131 & $54.8 \pm 11.1$ & M: 117/W: 16 & MI & $\begin{array}{c}\text { DS14 } \\
38.2\end{array}$ & $\begin{array}{l}\text { Anxiety: STAI, HAI } \\
\text { Depression: BDI }\end{array}$ & 7 \\
\hline $\begin{array}{l}\text { Bunevicius et al. } \\
2013 \text { [A2] }\end{array}$ & Netherlands & CS & 543 & $57.9 \pm 8.9$ & M: 388/W: 155 & CAD & $\begin{array}{l}\text { DS14 } \\
33.6\end{array}$ & $\begin{array}{l}\text { Anxiety: HADS-A } \\
\text { Depression: HADS-D }\end{array}$ & 6 \\
\hline $\begin{array}{l}\text { Cha et al. } \\
2013 \text { [A3] }\end{array}$ & Korea & CS & 111 & NA & M: 62/W: 49 & $\begin{array}{c}\text { CAD } \\
\text { (Angina, MI) }\end{array}$ & $\begin{array}{c}\text { DS14 } \\
36.1\end{array}$ & $\begin{array}{l}\text { Anxiety: SCL-90R } \\
\text { Depression: SCL-90R } \\
\text { HRQoL: WHOQOL-BREF }\end{array}$ & 8 \\
\hline Choi, 2013 [A4] & Korea & CS & 240 & $66.8 \pm 8.2$ & M: 77/W: 163 & $\begin{array}{c}\text { CAD } \\
\text { (Angina, MI) }\end{array}$ & $\begin{array}{c}\text { DS14 } \\
38.3\end{array}$ & Depression: CES-D & 7 \\
\hline $\begin{array}{l}\text { Condén et al. } \\
2014 \text { [A5] }\end{array}$ & Sweden & PC & 946 & $70.1 \pm 11.8$ & M: 633/W: 313 & MI & $\begin{array}{c}\text { DS14 } \\
25.1\end{array}$ & $\begin{array}{l}\text { Anxiety: HADS-A } \\
\text { Depression: HADS-D }\end{array}$ & 7 \\
\hline $\begin{array}{l}\text { De Fazio et al. } \\
2012 \text { [A6] }\end{array}$ & Italy & CS & 70 & $63.4 \pm 13.2$ & M: 48/W: 22 & ACS & $\begin{array}{c}\text { DS14 } \\
76.0\end{array}$ & $\begin{array}{l}\text { Anxiety: HADS-A } \\
\text { Depression: HADS-D }\end{array}$ & 8 \\
\hline $\begin{array}{l}\text { Denollet et al. } \\
1998 \text { [A7] }\end{array}$ & Belgium & CS & 100 & $55.9 \pm 9.1$ & M: 90/W: 10 & $\begin{array}{c}\text { CHD } \\
\text { (MI, CABG, } \\
\text { PTCA) }\end{array}$ & $\begin{array}{c}\text { DS16 } \\
29.0\end{array}$ & $\begin{array}{l}\text { Anxiety: TMAS } \\
\text { Depression: BDI }\end{array}$ & 7 \\
\hline $\begin{array}{r}\text { Doyle et al. } \\
2011 \text { [A8] }\end{array}$ & Ireland & CS & 339 & $61.1 \pm 10.8$ & M: 295/W: 44 & ACS & $\begin{array}{c}\text { DS14 } \\
30.1\end{array}$ & $\begin{array}{l}\text { Anxiety: HADS-A } \\
\text { Depression: HADS-D, BDI }\end{array}$ & 7 \\
\hline $\begin{array}{l}\text { Kaur et al. } \\
2014 \text { [A9] }\end{array}$ & Malaysia & CS & 195 & $56.8 \pm 106$ & M: 144/W: 51 & CAD & $\begin{array}{c}\text { DS14 } \\
28.2\end{array}$ & $\begin{array}{l}\text { Anxiety: HADS-A } \\
\text { Depression: HADS-D }\end{array}$ & 7 \\
\hline $\begin{array}{l}\text { Kelpis et al. } \\
2013 \text { [A10] }\end{array}$ & Greece & CS & 323 & $65.1 \pm 9.0$ & M: 260/W: 63 & CAD & $\begin{array}{c}\text { DS14 } \\
18.2\end{array}$ & $\begin{array}{l}\text { Anxiety: HADS-A } \\
\text { Depression: HADS-D }\end{array}$ & 6 \\
\hline $\begin{array}{l}\text { Lambertus et al. } \\
2018 \text { [A11] }\end{array}$ & Germany & CS & 570 & $59.2 \pm 9.5$ & M: 450/W: 120 & CHD & $\begin{array}{c}\text { DS14 } \\
60.1\end{array}$ & $\begin{array}{l}\text { Depression: HADS-D, } \\
\text { HAM-D }\end{array}$ & 7 \\
\hline $\begin{array}{l}\text { Lee, } \\
\text { 2015 [A12] }\end{array}$ & Korea & PC & 84 & $57.5 \pm 7.8$ & M: 65/W: 19 & $\begin{array}{c}\text { CAD } \\
\text { (Angina, MI) }\end{array}$ & $\begin{array}{c}\text { DS14 } \\
31.0\end{array}$ & $\begin{array}{l}\text { Depression: BDI } \\
\text { HRQoL: SF-12 }\end{array}$ & 7 \\
\hline $\begin{array}{l}\text { Lee et al. } \\
2018 \text { [A13] }\end{array}$ & Korea & CS & 63 & $59.8 \pm 11.1$ & M: 44/W: 19 & ACS & $\begin{array}{c}\text { DS14 } \\
33.3\end{array}$ & $\begin{array}{l}\text { Anxiety: HADS-A } \\
\text { Depression: HADS-D }\end{array}$ & 7 \\
\hline $\begin{array}{l}\text { Lim et al. } \\
2011 \text { [A14] }\end{array}$ & Korea & CS & 111 & $61.4 \pm 10.6$ & M: 70/W: 41 & CHD & $\begin{array}{c}\text { DS14 } \\
27.0\end{array}$ & $\begin{array}{l}\text { Anxiety: STAI } \\
\text { Depression: CES-D } \\
\text { HRQoL: GHQ }\end{array}$ & 6 \\
\hline $\begin{array}{l}\text { Lin et al. } \\
2016 \text { [A15] }\end{array}$ & Taiwan & CS & 168 & $61.3 \pm 7.3$ & M: 145/W: 23 & CAD & $\begin{array}{c}\text { DS14 } \\
25.0\end{array}$ & $\begin{array}{l}\text { Anxiety: BAI } \\
\text { Depression:BDI }\end{array}$ & 6 \\
\hline $\begin{array}{r}\text { Middel et al. } \\
2014 \text { [A16] }\end{array}$ & Netherlands & PC & 198 & $64.9 \pm 9.9$ & M: 155/W: 43 & CHD & $\begin{array}{c}\text { DS14 } \\
11.6\end{array}$ & $\begin{array}{l}\text { Anxiety: HADS-A } \\
\text { Depression: HADS-D } \\
\text { HRQoL: SF-36 }\end{array}$ & 8 \\
\hline $\begin{array}{r}\text { Molloy et al. } \\
2008 \text { [A17] }\end{array}$ & $\begin{array}{l}\text { United } \\
\text { Kingdom }\end{array}$ & CS & 70 & $60.6 \pm 10.3$ & M: 58/W: 12 & ACS & $\begin{array}{l}\text { DS16 } \\
38.6\end{array}$ & Depression: BDI & 8 \\
\hline $\begin{array}{r}\text { Molloy et al. } \\
2012 \text { [A18] }\end{array}$ & $\begin{array}{l}\text { United } \\
\text { Kingdom }\end{array}$ & PC & 165 & $61.7 \pm 10.9$ & M: 142/W: 23 & ACS & $\begin{array}{c}\text { DS14 } \\
30.3\end{array}$ & Depression: BDI & 7 \\
\hline $\begin{array}{l}\text { Mommersteeg et al. } \\
2013 \text { [A19] }\end{array}$ & Netherlands & CS & 273 & $62.1 \pm 9.65$ & M: 133/W: 140 & CAD & $\begin{array}{c}\text { DS14 } \\
30.0\end{array}$ & $\begin{array}{l}\text { Anxiety: HADS-A } \\
\text { Depression: HADS-D } \\
\text { HRQoL: SAQ }\end{array}$ & 8 \\
\hline
\end{tabular}

$\mathrm{ACS}=$ acute coronary syndrome; $\mathrm{BAI}=$ beck anxiety inventory; $\mathrm{BDI}=$ beck depression inventory; $\mathrm{BSI}=$ brief symptom inventory; $\mathrm{CAD}=\mathrm{coronary}$ artery

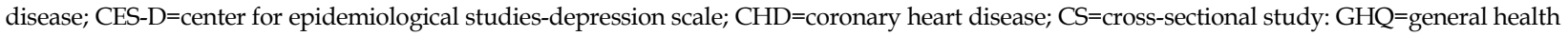
questionnaire/quality of life-12; HADS=Hospital anxiety depression scale; HAI=Health anxiety inventory; HAM-D=Hamilton depression rating scale; HRQL =health-related quality of life; MacNew=MacNew heart disease health-related quality of life instrument; MI=myocardial infarction; $\mathrm{MQ}=$ Masstrichit questionnaire; $\mathrm{NA}=$ not applicable; $\mathrm{PC}=$ prospective cohort study; $\mathrm{SAQ}=$ Seattle angina questionnaire; SCL-90R=symptom checklist-90-Revision; SF-12=medical outcomes study questionnaire short form 12; SF-36=medical outcomes study questionnaire short form 36; STAI=state-trait anxiety inventory; TMAS=Taylor manifest anxiety scale; W=women; WHOQOL-BREF=World Health Organization quality of life assessment instrument-BREF. 
Table 1. Descriptive Summary of Included Studies (Continued)

\begin{tabular}{|c|c|c|c|c|c|c|c|c|c|}
\hline Author & Country & Design & $\mathrm{n}$ & $\begin{array}{c}\text { Age } \\
\mathrm{M} \pm \mathrm{SD}\end{array}$ & $\begin{array}{c}\text { Gender } \\
\mathrm{n}\end{array}$ & Diagnosis & $\begin{array}{l}\text { Type D } \\
\text { Scale, \% }\end{array}$ & $\begin{array}{l}\text { Outcome } \\
\text { scale }\end{array}$ & $\begin{array}{c}\text { Quality } \\
\text { score }\end{array}$ \\
\hline $\begin{array}{l}\text { Ossola et al. } \\
2015 \text { [A20] }\end{array}$ & Italy & PC & 304 & $61.4 \pm 10.9$ & M: 245/W: 59 & ACS & $\begin{array}{l}\text { DS14 } \\
34.2\end{array}$ & $\begin{array}{l}\text { Anxiety: HADS-A } \\
\text { Depression: HADS-D }\end{array}$ & 8 \\
\hline $\begin{array}{l}\text { Park et al. } \\
2010 \text { [A21] }\end{array}$ & Korea & CS & 106 & NA & M: 83/W: 23 & CAD & $\begin{array}{c}\text { DS14 } \\
27.4\end{array}$ & $\begin{array}{l}\text { Anxiety: HADS-A } \\
\text { Depression: HADS-D } \\
\text { HRQoL: SAQ }\end{array}$ & 7 \\
\hline $\begin{array}{l}\text { Pedersen et al. } \\
2007 \text { [A22] }\end{array}$ & Netherlands & PC & 306 & $64.0 \pm 10.7$ & M: 150/W: 156 & CAD & $\begin{array}{l}\text { DS14 } \\
36.2\end{array}$ & $\begin{array}{l}\text { Anxiety: HADS-A } \\
\text { Depression: HADS-D }\end{array}$ & 8 \\
\hline $\begin{array}{l}\text { Pelle et al. } \\
2008 \text { [A23] }\end{array}$ & Netherlands & PC & 368 & $58.1 \pm 10.2$ & M: 290/W: 78 & CAD & $\begin{array}{l}\text { DS14 } \\
36.3\end{array}$ & $\begin{array}{l}\text { Anxiety: HADS-A } \\
\text { Depression: HADS-D } \\
\text { HRQoL: SF-36 }\end{array}$ & 7 \\
\hline $\begin{array}{l}\text { Smith et al. } \\
2008 \text { [A24] }\end{array}$ & Netherlands & PC & 401 & $62.9 \pm 10.9$ & M: 296/W: 105 & CHD & $\begin{array}{c}\text { DS14 } \\
32.3\end{array}$ & Depression: MQ & 8 \\
\hline $\begin{array}{l}\text { Son, } \\
2009 \text { [A25] }\end{array}$ & Korea & CS & 200 & $59.9 \pm 11.7$ & M: 99/W: 101 & $\begin{array}{c}\text { CAD } \\
\text { (Angina, MI) }\end{array}$ & $\begin{array}{c}\text { DS14 } \\
38.0\end{array}$ & $\begin{array}{l}\text { HRQoL: Korean version of } \\
\text { HRQoL }\end{array}$ & 7 \\
\hline $\begin{array}{l}\text { Spindler et al. } \\
2007 \text { [A26] }\end{array}$ & Netherlands & PC & 167 & $60.3 \pm 11.0$ & M: 102/W: 65 & CAD & $\begin{array}{l}\text { DS14 } \\
58.7\end{array}$ & $\begin{array}{l}\text { Anxiety: HADS-A } \\
\text { Depression: HADS-D }\end{array}$ & 8 \\
\hline $\begin{array}{l}\text { Svansdottir et al. } \\
2012 \text { [A27] }\end{array}$ & Iceland & PC & 268 & $62.9 \pm 10.5$ & M: 199/W: 69 & CAD & $\begin{array}{l}\text { DS14 } \\
26.1\end{array}$ & $\begin{array}{l}\text { Anxiety: HADS-A } \\
\text { Depression: HADS-D }\end{array}$ & 8 \\
\hline $\begin{array}{l}\text { van Gestel et al. } \\
2007 \text { [A28] }\end{array}$ & Netherlands & PC & 416 & $62.8 \pm 10.8$ & M: 312/W: 104 & Angina & $\begin{array}{c}\text { DS14 } \\
24.8\end{array}$ & $\begin{array}{l}\text { Anxiety: HADS-A } \\
\text { Depression: HADS-D }\end{array}$ & 8 \\
\hline $\begin{array}{l}\text { Vilchinsky et al. } \\
2011 \text { [A29] }\end{array}$ & Israel & PC & 94 & $56.3 \pm 7.6$ & M: 94/W: 0 & ACS & $\begin{array}{l}\text { DS14 } \\
22.3\end{array}$ & $\begin{array}{l}\text { Anxiety: BSI-A } \\
\text { Depression: BSI-D }\end{array}$ & 8 \\
\hline $\begin{array}{l}\text { Vukovic et al. } \\
2014 \text { [A30] }\end{array}$ & Serbia & CS & 79 & $53.9 \pm 8.5$ & M: 62/W: 17 & CAD & $\begin{array}{c}\text { DS14 } \\
34.2\end{array}$ & Depression: BDI & 7 \\
\hline
\end{tabular}

$\mathrm{ACS}=$ acute coronary syndrome; $\mathrm{BAI}=$ beck anxiety inventory; $\mathrm{BDI}=$ beck depression inventory; $\mathrm{BSI}=$ brief symptom inventory; $\mathrm{CAD}=\mathrm{coronary}$ artery disease; $\mathrm{CES}-\mathrm{D}=$ center for epidemiological studies-depression scale; $\mathrm{CHD}=$ coronary heart disease; $\mathrm{CS}=\mathrm{cross}-\mathrm{sectional}$ study: $\mathrm{GHQ}=\mathrm{general}$ health questionnaire/quality of life-12; HADS=Hospital anxiety depression scale; HAI=Health anxiety inventory; HAM-D=Hamilton depression rating scale; HRQoL=health-related quality of life; MacNew=MacNew heart disease health-related quality of life instrument; MI=myocardial infarction; $\mathrm{MQ}=$ Masstrichit questionnaire; $\mathrm{NA}=$ not applicable; $\mathrm{PC}=$ prospective cohort study; $\mathrm{SAQ}=$ Seattle angina questionnaire; $\mathrm{SCL}-90 \mathrm{R}=$ symptom checklist-90-Revision; SF-12=medical outcomes study questionnaire short form 12; SF-36=medical outcomes study questionnaire short form 36; STAI=state-trait anxiety inventory; TMAS=Taylor manifest anxiety scale; $\mathrm{W}=$ women; WHOQOL-BREF=World Health Organization quality of life assessment instrument-BREF.

덜란드에서 실시되었다. 연구대상자의 특성을 살펴보면, 11 편(35.5\%)의 연구가 100 199명의 대상자를 포함하였으 며 500명 이상의 대단위 연구는 3편이었다. 대상자의 평균연 령은 60 65세가 14편(48.3\%)로 가장 많았고, 남성의 비율이 $50 \sim 80 \%$ 인 연구가 18 편(58.1\%)를 차지하였으며, 급성관상동 맥증후군 환자만을 대상으로 한 연구가 7편(22.6\%), 관상동맥 질환자를 대상으로 한 연구는 15 편(48.4\%)이었다. D유형 성격 의 분포는 평균 $33.7 \%$ 으로 $11.6 \%$ 에서 $76.0 \%$ 까지 다양하였 고, $\mathrm{D}$ 유형 성격 측정도구는 DS14이 29편(93.5\%)으로 가장 많 았다. 결과변수 유형별로 우울은 29편(93.5\%), 불안은 22편 (71.0\%), 건강 관련 삶의 질은 9편(29.0\%)의 연구에서 측정되 었으며, 각각의 결과변수 측정에 다빈도로 사용된 도구는 우울
과 불안은 Hospital Anxiety and Depression Scale (HADS) 이었으며 건강 관련 삶의 질은 Medical Outcomes Study Questionnaire Short Form (SF)이었다(Tables 1, 2).

\section{D유형 성격의 효과크기}

메타분석 대상 논문 31 편 중 $\mathrm{D}$ 유형 성격에 따른 우울은 26 편, 불안은 17 편, 건강 관련 삶의 질을 신체적 안녕 및 정신적 안녕으로 구분하여 보고한 연구는 각 4 편에서 보고되었다. 변량효과모형을 이용하여 효과크기를 분석한 결과(Table 3, Figure 2), D유형 성격에 따른 우울의 효과크기 SMD는 0.92 (95\% CI=0.74 1.10, Z=10.30, $p<.001$ )이었고, 효과크기의 이 
Table 2. General Characteristics of Studies Included in Systematic Review and Meta-analysis

\begin{tabular}{|c|c|c|c|}
\hline \multicolumn{2}{|l|}{ Characteristics } & Categories & $\mathrm{n}(\%)$ \\
\hline \multirow{2}{*}{\multicolumn{2}{|c|}{ Publication year }} & $\leq 2010$ & $9(29.0)$ \\
\hline & & $\geq 2016$ & $4(12.9)$ \\
\hline \multirow{2}{*}{\multicolumn{2}{|c|}{ Publication country }} & Korea & $7(22.6)$ \\
\hline & & Others & $24(77.4)$ \\
\hline \multirow[t]{15}{*}{ Participants } & \multirow{4}{*}{$\begin{array}{l}\text { Number } \\
(\text { range }=63.0 \sim 946.0, \text { mean=245.2, } \text { median=195.0) }\end{array}$} & $\leq 99$ & $6(19.4)$ \\
\hline & & 100 199 & $11(35.5)$ \\
\hline & & $200 \sim 499$ & $11(35.5)$ \\
\hline & & $\geq 500$ & $3(9.6)$ \\
\hline & \multirow{3}{*}{$\begin{array}{l}\text { Mean age } \\
\quad(\text { range }=53.9 \sim 71.1, N=29)\end{array}$} & $\leq 59.9$ & $11(37.9)$ \\
\hline & & $60.0 \sim 64.9$ & $14(48.3)$ \\
\hline & & $\geq 65.0$ & $4(13.8)$ \\
\hline & \multirow{3}{*}{$\begin{array}{l}\% \text { of Male } \\
(\text { range=32.1 100.0, mean=72.8, median=75.0) }\end{array}$} & $\leq 49.9$ & $4(12.9)$ \\
\hline & & $50.0 \sim 79.9$ & $18(58.1)$ \\
\hline & & $\geq 80.0$ & $9(29.0)$ \\
\hline & \multirow[t]{5}{*}{ Diagnosis group } & Angina & $1(3.2)$ \\
\hline & & Acute coronary syndrome & $7(22.6)$ \\
\hline & & Coronary artery disease & $15(48.4)$ \\
\hline & & Coronary heart disease & $5(16.1)$ \\
\hline & & Myocardial infarction & $3(9.7)$ \\
\hline \multirow[t]{3}{*}{ Type D personality } & \multicolumn{3}{|l|}{$\begin{array}{l}\text { Prevalence } \\
(\text { range=11.6 76.0, mean=33.7, median=32.3) }\end{array}$} \\
\hline & \multirow[t]{2}{*}{ Measurement } & DS14 & $29(93.5)$ \\
\hline & & DS16 & $2(6.5)$ \\
\hline \multirow{14}{*}{$\begin{array}{l}\text { Outcome \& } \\
\text { measurement }\end{array}$} & \multirow[t]{5}{*}{ Depression } & & $29(93.5)$ \\
\hline & & BDI & $8(27.6)$ \\
\hline & & CES-D & $2(6.9)$ \\
\hline & & HADS & $17(58.6)$ \\
\hline & & Others & $2(6.9)$ \\
\hline & \multirow[t]{4}{*}{ Anxiety } & & $22(71.0)$ \\
\hline & & HADS & $16(72.7)$ \\
\hline & & STAI & $2(9.1)$ \\
\hline & & Others & $4(18.2)$ \\
\hline & \multirow[t]{5}{*}{ Health-related quality of life } & & $9(29.0)$ \\
\hline & & SAQ & $2(22.3)$ \\
\hline & & SF-12, SF-36 & $3(33.3)$ \\
\hline & & MacNew & 1 (11.1) \\
\hline & & Others & $3(33.3)$ \\
\hline
\end{tabular}

$\mathrm{BDI}=$ beck depression inventory; $\mathrm{CES}-\mathrm{D}=$ center for epidemiological studies-depression scale; $\mathrm{DS} 14=14$ item of type D personality scale; DS16=16 item of type D personality scale; HADS=Hospital anxiety depression scale; MacNew=MacNew heart disease health-related quality of life instrument; SAQ=Seattle angina questionnaire; SF-12=medical outcomes study questionnaire short form 12; SF-36=medical outcomes study questionnaire short form 36; STAI=state-trait anxiety inventory; ${ }^{\dagger}$ Multiple response.

질성 $\mathrm{I}^{2}$ 은 $84.7 \%(\mathrm{Q}=163.70, \mathrm{df}=25, p<.001)$ 으로 큰 편이었다

(Figure 2-A). 다음으로 D유형 성격에 따른 불안의 효과크기 $\mathrm{SMD}$ 는 1.19 (95\% CI=0.81 1.57, Z=6.10, $p$ <.001)이었고, 효 과크기의 이질성은 $\mathrm{I}^{2}$ 은 $95.3 \%(\mathrm{Q}=342.70, \mathrm{df}=16, p<.001)$ 으 로 큰 편이었다(Figure 2-B). 다음으로 D유형 성격에 따른 건 강 관련 삶의 질 중 신체적 안녕의 효과크기 $\mathrm{SMD}$ 는 -0.56
(95\% CI=-0.75 -0.38, Z=-6.04, $p<.001$ ), 정신적 안녕의 효과 크기 SMD는 -0.91 (95\% CI=-1.10 -0.73, Z=-9.69, $p<.001)$ 이었고, 효과크기의 이질성 $\mathrm{I}^{2}$ 은 각 $1.6 \%(\mathrm{Q}=3.05, \mathrm{df}=3, p=$ $.384)$ 와 $0.0 \%(\mathrm{Q}=0.50, \mathrm{df}=3, p=.920)$ 으로 작거나 없었다

(Figure 2-C, 2-D). 


\begin{tabular}{|c|c|}
\hline Study name & Qutcome \\
\hline AnnagUR (2016) & $\mathrm{BDI}$ \\
\hline Bunevicius (2013) & BDI \\
\hline Cha (2013) & SCL-90R \\
\hline Choi (2013) & CES-D \\
\hline Conden (2014) & HADS \\
\hline Defazuo (2012) & HADS \\
\hline Denollet (1998) & $\mathrm{BDI}$ \\
\hline Doyle (2011) & HADS \\
\hline Kaur (2014) & HADS \\
\hline Kelpis (2013) & HADS \\
\hline Lambertus (2018) & HADS \\
\hline Lee KH (2015) & BDI \\
\hline Lee SJ (2018) & HADS \\
\hline Lim (2011) & CES-D \\
\hline $\operatorname{Lin}(2016)$ & $\mathrm{BDI}$ \\
\hline Middel (2014) & HADS \\
\hline Molloy (2008) & BDI \\
\hline Molloy et al (2012) & BDI \\
\hline Mommersteeg (2013) & HADS \\
\hline Ossola (2015) & HADS \\
\hline Park (2010) & HADS \\
\hline Pedersen (2007) & HADS \\
\hline Smith (2008) & Masstricht questionnaire \\
\hline Svansdottir (2012) & HADS \\
\hline Vilchinsky (2011) & Brief symptom inventor \\
\hline Vukovic (2014) & $\mathrm{BDI}$ \\
\hline
\end{tabular}

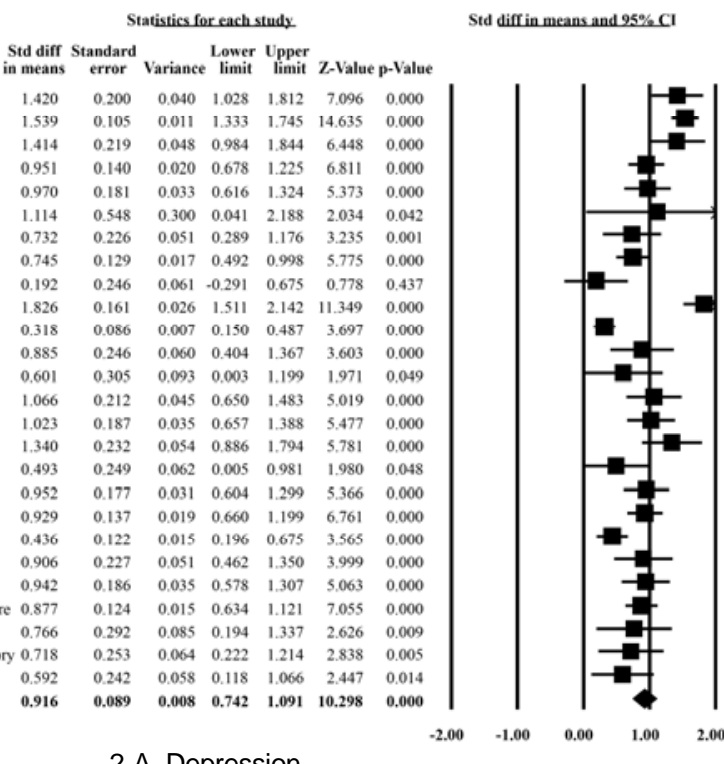

\begin{tabular}{|c|c|}
\hline Study name. & Outcome. \\
\hline AnnaguR (2016) & HAI \\
\hline Bunevicius (2013) & HADS \\
\hline Cha (2013) & SCL-90R \\
\hline Defazuo (2012) & HADS \\
\hline Kaur (2014) & HADS \\
\hline Kelpis (2013) & HADS \\
\hline Lambertus (2018) & HADS \\
\hline Lee SJ (2018) & HADS \\
\hline $\operatorname{Lim}(2011)$ & STAI \\
\hline $\operatorname{Lin}(2016)$ & BAI \\
\hline Middel (2014) & HADS \\
\hline Mommersteeg (2013) & HADS \\
\hline Park (2010) & HADS \\
\hline Spinder (2007) & HADS \\
\hline Svansdottir (2012) & HADS \\
\hline van Gestel (2007) & HADS \\
\hline Vilchinsky (2011) & Brief sy \\
\hline
\end{tabular}

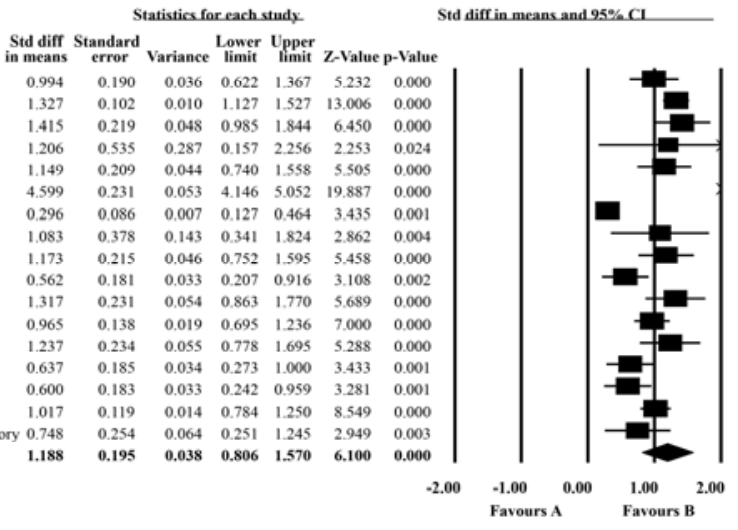

2-B. Anxiety

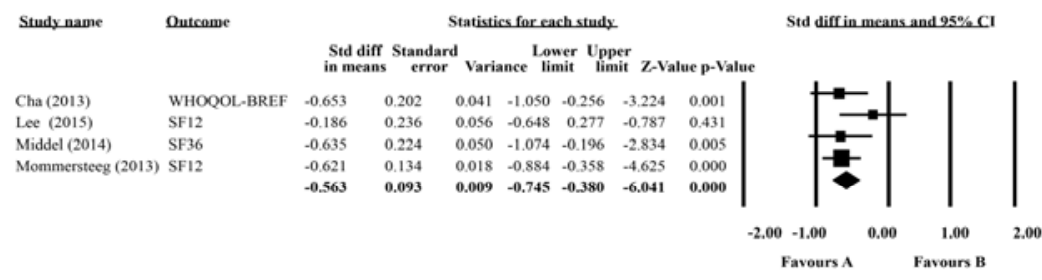

2-C. Health-related quality of life: physical

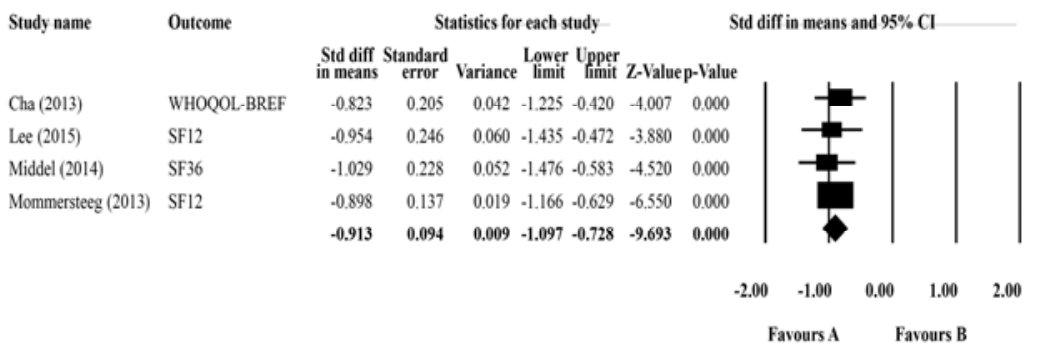

2-D. Health-related quality of life: mental

Figure 2. Forest plot of depression, anxiety, and health-related quality of life. 
Table 3. Meta-analysis of the Impact of Type D personality on Depression, Anxiety, and Health-related Quality of Life in Patients with Coronary Artery Disease

$(N=3,417)$

\begin{tabular}{|c|c|c|c|c|c|c|c|c|c|c|c|c|}
\hline \multirow{2}{*}{ Variables } & \multirow{2}{*}{$\mathrm{k}$} & \multirow{2}{*}{$\mathrm{n}$} & \multicolumn{3}{|c|}{ Homogeneity test } & \multirow{2}{*}{$\begin{array}{c}\text { Overall } \\
\text { effect }\end{array}$} & \multirow{2}{*}{$95 \% \mathrm{CI}$} & \multirow{2}{*}{ z } & \multirow{2}{*}{$p$} & \multicolumn{2}{|c|}{ ER } & \multirow{2}{*}{$\mathrm{FSN}^{\dagger}$} \\
\hline & & & Q & $\mathrm{df}$ & $p$ & & & & & $\mathrm{t}$ & $p$ & \\
\hline Depression & 26 & 6,419 & 163.70 & 25 & $<.001$ & 0.92 & $0.74 \sim 1.10$ & 10.30 & $<.001$ & 0.69 & .429 & 4,958 \\
\hline Anxiety & 17 & 4,308 & 342.70 & 16 & $<.001$ & 1.19 & $0.81 \sim 1.57$ & 6.10 & $<.001$ & 1.47 & .162 & 2,766 \\
\hline HRQoL: physical & 4 & 666 & 3.05 & 3 & .384 & -0.56 & $-0.75 \sim-0.38$ & -6.04 & $<.001$ & 0.82 & .499 & 31 \\
\hline HRQoL: mental & 4 & 666 & 0.50 & 3 & .920 & -0.91 & $-1.10 \sim-0.73$ & -9.69 & $<.001$ & 0.60 & .734 & 90 \\
\hline
\end{tabular}

$\mathrm{CI}=$ confidence interval; $\mathrm{ER}=$ Egger's regression; $\mathrm{FSN}=$ Fail-safe number; $\mathrm{HRQoL}=$ health-related quality of life; $\mathrm{k}=$ number of studies; ${ }^{\dagger} \mathrm{FSN}$ was analyzed using Orwin method.

\section{5. 효과크기의 이질성 검증}

효과크기의 이질성이 높았던 우울과 불안에 대한 추가적인 설명을 위해, 연구의 일반적 특성인 출판년도, 연구수행 지역 $(0=$ 국외, $1=$ 국내 $)$, 연구대상자의 수, 진단명 $(0=$ 기타, $1=$ 관상 동맥질환), 질평가 점수를 조절변수로 선정하여 효과크기의 이질성을 설명하기 위해 메타회귀분석을 실시하였다. 그 결 과, 출판년도가 증가할수록 우울의 효과크기 $(\mathrm{Z}=-2.00, p=.045)$ 와 불안의 효과크기 $(\mathrm{Z}=-4.74, p<.001)$ 가 통계적으로 유의 하게 감소하였으며(Figure 3-A, 3-C), 질평가 점수가 증가 할수록 우울의 효과크기 $(\mathrm{Z}=-2.83, p=.005)$ 와 불안의 효과크 기 $(\mathrm{Z}=-3.52, p<.001)$ 가 통계적으로 유의하게 감소하였다 (Figure 3-B, 3-D). 그리고 진단명과 관련하여 다른 심혈관질 환자들 보다 관상동맥질환자만을 대상으로 수행하였을 때 우 울의 효과크기 $(Z=3.44, p=.001)$ 가 통계적으로 유의하게 증가 하였다.

\section{6. 비뚤림 검정 결과}

깔때기 도표로 대칭정도를 확인한 후, Egger's regression test로 비대칭 정도가 통계적으로 유의한지 여부를 확인하였 다. 그 결과 우울 $(p=.429)$, 불안 $(p=.162)$, 신체적 $(p=.499)$ 및 정신적 건강 관련 삶의 질 $(p=.734)$ 는 비대칭 정도가 통계적으 로 유의하지 않았다. 한편 출판비뚤림 평가에서 안전계수는 우 울 4,958편, 불안 2,766편, 신체적 안녕 31편 및 정신적 안녕 90 편으로 신체적 안녕을 제외한 우울, 불안, 정신적 안녕의 안전 계수는 '충분히 큰 것'으로 나타났다.

\section{논 의}

본 연구는 체계적 문헌고찰과 메타분석을 통해 관상동맥질
환 환자에서 $\mathrm{D}$ 유형 성격이 우울, 불안과 건강 관련 삶의 질에 미치는 영향을 규명하고자 수행되었다. 이를 위해 총 1,128 편 의 연구들이 검색되었고 이 중 선정 및 배제기준에 따라 31 개의 연구가 최종적으로 선택되었다.

분석에 포함된 31편 대부분이 2011년 이후에 수행된 것으 로, 이는 관상동맥질환 환자의 경과 및 예후에 영향을 미치는 주요 요인으로서 $\mathrm{D}$ 유형 성격에 대한 관심이 부각되고 있음을 보여준다. 그러나 31 편 중 24편이 네덜란드를 비롯한 국외에서 이루어졌고, 국내에서는 7편의 연구만이 보고되었다. 국외의 경우 관상동맥질환의 발생 및 예후에 영향을 미치는 주요 요인 으로 $\mathrm{D}$ 유형 성격이 대두되면서 이와 관련된 다양한 연구들이 수행되고 있다 $[1,3,4]$. 이에 반해 국내에서는 관상동맥질환에 서 $\mathrm{D}$ 유형 성격이 건강문제나 예후에 미치는 영향이나 관련성 에 대한 연구가 부족한 실정으로 지속적으로 $\mathrm{D}$ 유형 성격과 이 차적인 건강문제, 삶의 질이나 예후와의 관련성에 대한 연구들 이 수행되어야 할 것이다. 다음으로 총 31 개 연구의 연구대상 자 수는 최소 63명에서 최대 946명으로 중앙값 195 명이었으며 500 명 이상을 대상으로 한 연구도 3편이었다. 한편 연구대상자 의 성별 비율은 전 세계적으로 남성의 관상동맥질환의 유병률 이 여성보다 높게 보고되고 있기 때문에 남성이 여성보다 3 배 가량 많았다. 그러나 성별에 따른 $\mathrm{D}$ 유형 성격의 빈도간의 차이 가 있는지 파악하기 위해서 추후 여성의 비율을 증가하여 대단 위의 연구를 수행하는 것이 요구된다.

다음으로 연구결과 변수로 우울과 불안을 측정한 연구는 각 29편과 22편인데 반해 건강 관련 삶의 질을 측정한 연구는 31 편 중 9편(29.0\%)에 불과하였다. 관상동맥질환에서 $\mathrm{D}$ 유형 성 격이 가지는 영향을 살펴본 메타분석들은 주로 심인성 혹은 비 심인성 사망비나 MACE의 발생비를 보고한 것[1-3]이 대부분 으로, 건강 관련 삶의 질과 같은 환자보고형 결과를 보고한 연 구는 부족하다. 그러나 최근 관상동맥질환과 같은 만성질환에 서 환자보고형 결과가 사망률과 질병증상 악화에 따른 재입원 


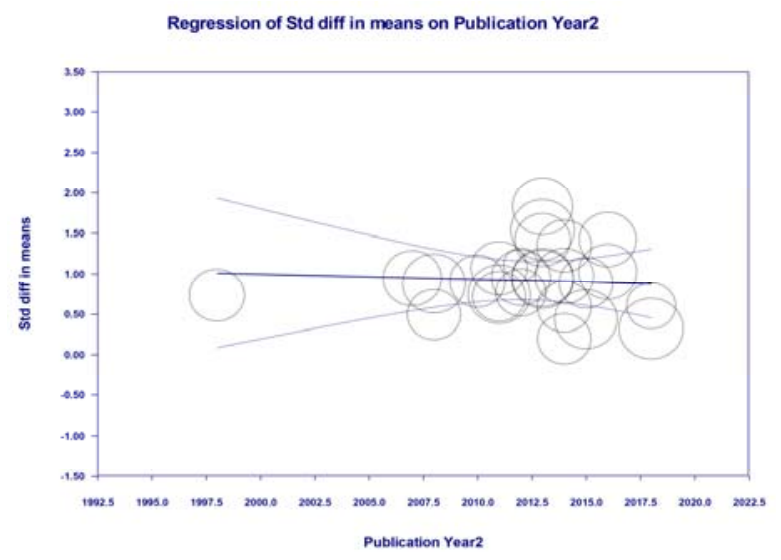

3-A. Publication year on depression

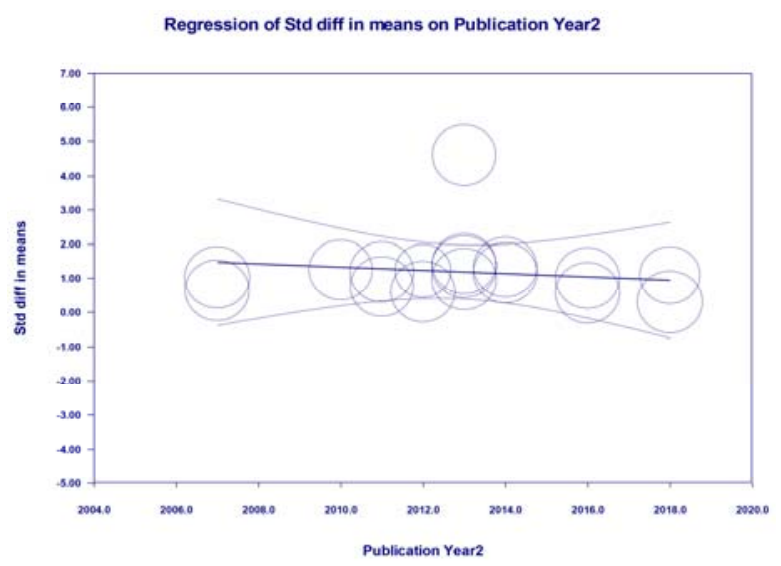

3-C. Publication year on anxiety

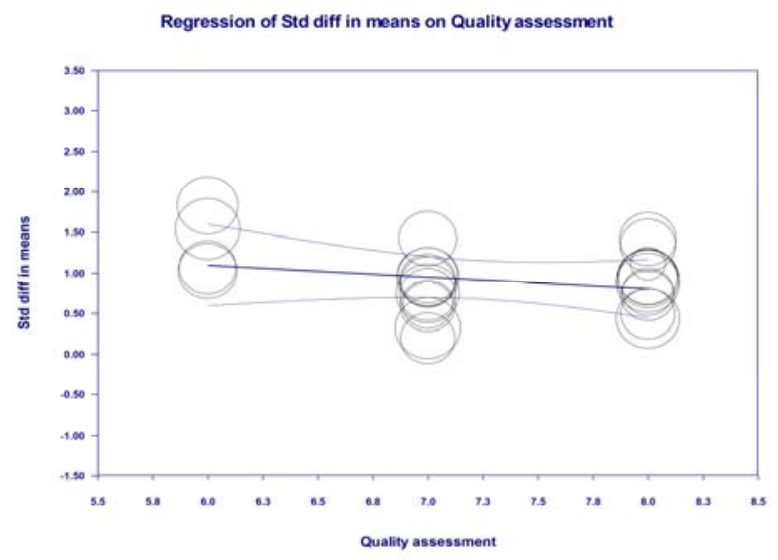

3-B. Quality score on depression

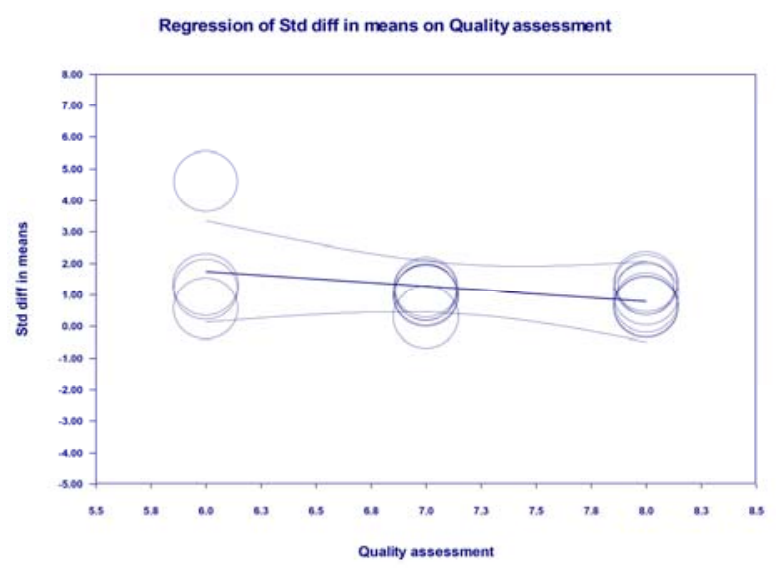

3-D. Quality score on anxiety

Figure 3. Regression plot.

율 증가와 관련이 있는 것으로 보고되고 있으므로[16], 추후 인 지된 건강상태와 건강 관련 삶의 질과 $\mathrm{D}$ 유형 성격에 대한 연구 들이 계속적으로 수행되어야 할 것이다.

한편 31편 연구에서 사용된 측정도구를 분석한 결과, $\mathrm{D}$ 유형 성격은 DS14으로 측정한 연구가 대부분이었다. DS14는 현재 22 개국에서 횡문화적 타당도가 입증되어 다양한 연구에 활용 되고 있는 도구로[24], 국내에서도 Lim 등[A14]이 한국 고유 의 문화적, 사회적 특성을 고려하여 수정·보완한 한국어판 DS14가 대부분 D유형 성격과 관련된 연구들에서 활용되고 있 다. 그러나 여전히 국내 심혈관질환자 이외의 만성질환자를 대 상으로 한국어판 DS14의 타당도와 신뢰도를 확인한 연구는 소 수에 지나지 않으므로 심혈관질환자 뿐 아니라 건강인이나 혹 은 다른 만성질환을 가진 대상자를 대상으로 DS14의 타당도를 검증하는 연구들이 요구된다. 우울과 불안의 경우 관상동맥질 환자에서 타당도와 신뢰도가 입증된 도구인 HADS를 가장 빈 번하게 사용하고 있었다[25,A9]. 그러나 HADS는 심혈관질환
자를 대상으로 개발된 도구가 아니므로 심혈관질환자가 경험 하는 심리적 디스트레스의 핵심 속성이 반영된 특이적 도구 개 발이 요구된다. 다음으로 건강 관련 삶의 질의 측정도구를 보면 대부분이 일반적 건강 관련 삶의 질 측정도구를 사용하고 있었 으며 질병특이적 도구를 사용한 연구는 단 2편이었다. 일반적 평가 도구는 광역의 집단을 대상으로 개발되어 다양한 집단에 서 그 사용이 용이하다는 장점이 있으나 질병특이적 도구는 심 질환자를 대상으로 개발되었기 때문에 심질환별 다양한 증상 경험을 이해하고 질병 및 증상으로 인한 신체적, 정서적, 사회 적, 기능적인 삶의 질의 수준을 파악하는데 도움이 된다[26,27]. 그러므로 추후 국내 심질환자의 건강 관련 삶의 질 수준을 이해 하고 나아가 $\mathrm{D}$ 유형 성격에 따른 건강상태의 변화나 차이를 충 분히 이해하기 위해서 질병특이적 건강 관련 삶의 질 도구를 사 용한 추후 연구들이 반복적으로 수행되어야 할 것이다.

선정된 연구를 중심으로 질 평가를 실시한 결과 JBI [23]의 평가항목 8 개 중 6 개 이상을 만족하고 있어 전반적인 질적 평가 
점수는 양호한 것으로 나타났다. 그러나 대부분의 연구들이 혼 동변수에 대한 설명 또는 고려가 부족하였고, 포함된 연구들 중 일부가 다기관의 추적조사연구자료를 활용하여 이차분석한 것으로 혼동변수를 통제하는 통계분석법이 적절히 이루어지 지 않았다는 제한점을 가진다.

메타분석 결과를 보면, $\mathrm{D}$ 유형 성격을 가진 관상동맥질환자 들은 $\mathrm{D}$ 유형 성격이 아닌 환자들에 비해 우울과 불안의 수준이 유의하게 높은 것으로 나타났다. 이는 D유형 성격의 환자들은 그렇지 않은 환자들에 비해 우울, 불안 등의 정서적 건강문제를 많이 가지는 것으로 알려져 있다고 보고한 선행연구결과와 일 치한다[5,6,28,A27,A28]. 그리고 본 연구에서 분석된 효과크 기를 다시 승산비로 재분석하여 ICD 삽입 환자를 대상으로 D 유형 성격인 환자에서 우울과 불안의 승산비를 메타분석한 선 행연구[7]와 비교한 결과, $\mathrm{D}$ 유형 성격의 관상동맥질환자는 우 울은 4.6 배, 불안은 4.3 배 발생비가 높은 것으로 확인되었다. 이 는 선행연구[7]에서 우울은 5.9 배, 불안은 4.0 배 높은 승산비로 발생한다고 보고한 연구결과와 유사한 결과이다. 따라서 $\mathrm{D}$ 유 형 성격을 고위험군으로 선별하고 우울과 불안과 같은 정서적 건강문제가 발생하거나 악화되지 않도록 심리적 지지 및 상담 이 주축이 되는 간호중재를 개발하여 제공하여야 할 것이다. 그 러나 $\mathrm{D}$ 유형 성격의 부정적 정서는 심리적 증상과 구분하기 어 렵고[2], D유형 성격과 우울 또는 불안이 동시에 있는 환자의 경우 심질환 재발이나 사망률이 높은 것으로 알려져 있음에도 $[2,5,6,27]$, 현재는 성격유형과 심리적 요인 간의 인과관계를 규 명한 연구가 미흡한 실정이다. 따라서 성격유형과 심리적 요인 이 어떠한 기전으로 심장병리학적 발생기전과 관련되었는지 를 규명하기 위하여 환자보고형 결과뿐 아니라 생행동지표를 이용한 후속연구들이 시도되어야 할 것이다.

다음으로 건강 관련 삶의 질에 대한 영향을 메타분석한 결 과, $\mathrm{D}$ 유형 성격을 가진 관상동맥질환자는 신체적 안녕 및 정신 적 안녕의 수준이 유의하게 낮은 것으로 나타났다. 본 연구에서 분석된 효과크기를 다시 승산비로 재분석하여 심혈관질환자 에서 $\mathrm{D}$ 유형 성격과 건강상태 손상과의 관계를 메타분석한 선 행연구[16]와 비교한 결과, $\mathrm{D}$ 유형 성격의 관상동맥질환자는 건강 관련 삶의 질 중 신체적 손상은 2.8 배, 정신적 손상은 4.8 배 승산비가 높은 것으로 확인되었다. 이는 선행연구[16]에서 신 체적 손상은 2.0 배, 정신적 손상은 2.5 배 높은 승산비를 보인다 고 보고한 연구결과보다 높은 수치이다. 비록 선행연구가 관상 동맥질환 외에 심부전과 말초혈관질환자들을 대상으로 한 연 구까지 분석에 포함하여 본 연구보다 대상자의 이질성이 높기 는 하지만, $\mathrm{D}$ 유형 성격이 신체적 안녕 및 정신적 안녕에 부정
적 영향을 미친다는 결과에서는 일치한다. $\mathrm{D}$ 유형 성격을 가진 사람은 상황을 부정적으로 바라보기 때문에 질병인식이 나쁘 고 질병으로 인한 증상을 더 심각하게 받아들이는 경향이 있는 반면 스트레스 상황을 개선하기 위해 적절한 대처기전을 사용 하지 못하는 경향이 있다 $[1,13,16]$. 건강인을 대상으로 D성격 유형에 대한 연구를 체계적 문헌고찰한 결과[19]에서도 $\mathrm{D}$ 유형 성격을 가진 사람은 신체화 증상을 많이 호소하고 감기증상을 더 많이 느끼며 건강 관련 삶의 질에 부정적 영향을 미친다고 보고하여 본 연구결과를 지지한다. 그러나 본 연구에 포함된 연 구들은 다양한 도구로 건강 관련 삶의 질을 측정하였고, 일부 연구에서만 건강 관련 삶의 질 하부영역의 통계치를 제시하였 기 때문에 건강 관련 삶의 질의 하부영역에 대한 분석을 수행할 수 없었다. 그러나 추후에는 축적된 후속연구의 근거를 토대로 심질환자의 전반적인 삶의 질에 부정적인 영향을 미칠 수 있는 협심통, 호흡곤란, 피로와 같은 주관적 건강문제들과 운동시의 제한점, 활동제한과 같은 기능적인 신체적 건강문제들, 질병으 로 인한 사회적 기능저하 등 다양한 하위영역에 대한 $\mathrm{D}$ 유형 성 격에 따른 차이를 확인해보는 체계적 문헌고찰 및 메타분석 연 구가 요구된다.

마지막으로 조절효과분석을 통해서 출판년도와 질평가 점 수, 그리고 연구대상자의 진단명에 관한 의미 있는 결과가 나타 났다. 이는 최근의 근거기반간호를 위한 연구방법론의 내외적 타당도가 강화되는 연구환경과 관련될 것이다. 즉 연구목적에 부합하는 적절한 대상자수의 모집, 신뢰도와 타당도가 검증된 측정도구의 사용 및 혼동변수를 고려한 통계 방법 등을 통해서 연구결과의 과잉추정이 발생하지 않았을 것으로 사료된다.

본 연구는 D유형 성격이 우울, 불안과 건강 관련 삶의 질과 같은 환자보고형 결과에 미치는 영향을 종합하여 분석했다는 점에서 연구의 의의가 있으나 다음과 같은 제한점으로 해석하 는데 주의가 필요하다. 첫째, 본 연구에서는 한국어와 영어로 출간된 논문, 그리고 $\mathrm{D}$ 유형 성격에 따른 우울, 불안과 건강 관 련 삶이 질을 보고한 조사연구만을 중점으로 하여 검색하여 선 별하였기 때문에 다소 제한된 논문이 메타분석에 포함되었다 는 제한점을 가진다. 둘째, 본 연구에서는 $\mathrm{D}$ 유형 성격과 우울, 불안이 동일한 시점에서 측정된 값만을 메타분석에 활용하였 기 때문에 이들 변수가 $\mathrm{D}$ 유형 성격과 건강 관련 삶의 질과의 관 계에서 매개 및 조절변수로서의 역할을 탐색하기 어렵다. 셋 째, 건강 관련 삶의 질의 경우 상대적으로 적은 4 편의 연구만이 메타분석에 포함되어 편견 가능성이 있을 수 있으므로 $\mathrm{D}$ 유형 성격과 건강 관련 삶의 질의 관련성에 대한 계속적인 추후연구 가 요구된다. 넷째, 본 연구는 검색엔진을 이용하여 수집된 연 
구를 대상으로 체계적 문헌고찰과 효과크기 계산을 실시하였 기 때문에, 미발표된 연구의 배제와 관련된 출판연구의 편견가 능성이 있다.

\section{결론 및 제언}

본 연구는 관상동맥질환 환자를 중심으로 $\mathrm{D}$ 유형 성격이 우 울, 불안과 건강 관련 삶의 질에 미치는 영향을 확인하기 위하 여 선행연구들을 체계적으로 고찰하고 질적 평가를 통해 선정 된 연구를 중심으로 메타분석을 실시하여 그 효과크기를 분석 하였다. 그 결과 관상동맥질환 환자에서 $\mathrm{D}$ 유형 성격은 우울과 불안의 수준을 높이고, 건강 관련 삶의 질의 수준을 저하시키는 것으로 나타났다. 의료수준의 발전에 따라 관상동맥질환을 가 진 만성질환자가 증가하고 있는 점을 고려할 때, 질병의 발생 및 예후와 관련된 위험요인인 D유형 성격의 효과크기를 산출 하였다는 점에서 본 연구의 의의가 있다. 본 연구의 결과를 토 대로 관상동맥질환 환자를 위한 간호중재를 제공할 때 $\mathrm{D}$ 유형 성격을 가진 사람들을 우선적으로 선별하여 우울과 불안을 완 화하여 건강수준을 향상시킬 수 있는 차별화된 간호중재를 제 공하는 것이 고려되어야 할 것이다.

\section{CONFLICTS OF INTEREST}

The authors declared no conflict of interest.

\section{AUTHORSHIP}

Study conception and design acquisition - BSH and PJ-H; Data collection - BSH and PJ-H; Analysis and interpretation of the data $\mathrm{BSH}$; Drafting and critical revision of the manuscript - BSH and PJ-H.

\section{ACKNOWLEDGEMENT}

This work has supported by the National Research Foundation of Korea (NRF) grant funded by the Korea government (MSIT) (No. NRF-2018R1D1A1B07043306).

\section{REFERENCES}

1. Denollet J. DS14: standard assessment of negative affectivity, social inhibition, and Type D personality. Psychosomatic Medicine. 2005;67(1):89-97.

https://doi.org/10.1097/01.psy.0000149256.81953.49

2. Grande G, Romppel M, Barth J. Association between Type D personality and prognosis in patients with cardiovascular dis- eases: a systematic review and meta-analysis. Annals of Behavioral Medicine. 2012;43(3):299-310.

https://doi.org/10.1007/s12160-011-9339-0

3. Kupper N, Denollet J. Type D personality as a risk factor in coronary heart disease: a review of current evidence. Current Cardiology Reports. 2018;20(11):104. https://doi.org/10.1007/s11886-018-1048-x

4. Piepoli MF, Hoes AW, Agewall S, Albus C, Brotons C, Catapano AL, et al. 2016 European guidelines on cardiovascular disease prevention in clinical practice: the sixth joint task force of the European society of cardiology and other societies on cardiovascular disease prevention in clinical practice (constituted by representatives of 10 societies and by invited experts) developed with the special contribution of the European association for cardiovascular prevention \& rehabilitation (EACPR). Atherosclerosis. 2016;252:207-74.

https://doi.org/10.1016/j.atherosclerosis.2016.05.037

5. Doyle F, McGee H, Delaney M, Motterlini N, Conroy R. Depressive vulnerabilities predict depression status and trajectories of depression over 1 year in persons with acute coronary syndrome. General Hospital Psychiatry. 2011;33(3):224-31. https://doi.org/10.1016/j.genhosppsych.2011.03.008

6. Lambertus F, Herrmann-Lingen C, Fritzsche K, Hamacher S, Hellmich M, Junger J, et al. Prevalence of mental disorders among depressed coronary patients with and without Type D personality. Results of the multi-center SPIRR-CAD trial. General Hospital Psychiatry. 2018;50:69-75. https://doi.org/10.1016/j.genhosppsych.2017.10.001

7. Starrenburg AH, Kraaier K, Pedersen SS, van Hout M, Scholten M, van der Palen J. Association of psychiatric history and Type D personality with symptoms of anxiety, depression, and health status prior to ICD implantation. International Journal of Behavioral Medicine. 2013;20(3):425-33.

https://doi.org/10.1007/s12529-012-9244-3

8. Park J-H, Bae SH. A systematic review of psychological distress as a risk factor for recurrent cardiac events in patients with coronary artery disease. Journal of Korean Academy of Nursing. 2011;41(5):704-14. https://doi.org/10.4040/jkan.2011.41.5.704

9. Song EK, Son YJ. The analysis of Type D personality research as a psychosocial risk factor in cardiovascular disease for elders with a chronic disease. Journal of Korean Academy of Nursing. 2008;38(1):19-28. https://doi.org/10.4040/jkan.2008.38.1.19

10. Barth J, Schumacher M, Herrmann-Lingen C. Depression as a risk factor for mortality in patients with coronary heart disease: a meta-analysis. Psychosomatic Medicine. 2004;66(6): 802-13. https://doi.org/10.1097/01.psy.0000146332.53619.b2

11. Roest AM, Martens EJ, Denollet J, de Jonge P. Prognostic association of anxiety post myocardial infarction with mortality 
and new cardiac events: a meta-analysis. Psychosomatic Medicine. 2010;72(6):563-9.

https://doi.org/10.1097/PSY.0b013e3181dbff97

12. Jo EH, Han SH, Lee MH, Kim SR. Influencing effects of Type D personality on symptom experiences and quality of life in patients with percutaneous coronary intervention. Korean Journal of Adult Nursing. 2016;28(5):536-45. https://doi.org/10.7475/kjan.2016.28.5.536

13. Schiffer AA, Denollet J, Widdershoven JW, Hendriks EH, Smith ORF. Failure to consult for symptoms of heart failure in patients with a type-D personality. Heart. 2007;93(7):814-8. https://doi.org/10.1136/hrt.2006.102822

14. Noh I-K, Kim MS. Moderating effect of lifestyle and Type D personality on the relationship between metabolic syndrome and severity of coronary artery disease. Korean Journal of Adult Nursing. 2018;30(3):290-300. https://doi.org/10.7475/kjan.2018.30.3.290

15. Sajobi TT, Wang M, Awosoga O, Santana M, Southern D, Liang Z, et al. Trajectories of health-related quality of life in coronary artery disease. Circulation: Cardiovascular Quality and Outcomes. 2018;11(3):e003661. https://doi.org/10.1161/circoutcomes.117.003661

16. Versteeg H, Spek V, Pedersen SS, Denollet J. Type D personality and health status in cardiovascular disease populations: a meta-analysis of prospective studies. European Journal of Preventive Cardiology. 2012;19(6):1373-80. https://doi.org/10.1177/1741826711425338

17. O'Dell KR, Masters KS, Spielmans GI, Maisto SA. Does type-D personality predict outcomes among patients with cardiovascular disease? A meta-analytic review. Journal of Psychosomatic Research. 2011;71(4):199-206.

https://doi.org/10.1016/j.jpsychores.2011.01.009

18. Mols F, Denollet J. Type D personality among noncardiovascular patient populations: a systematic review. General Hospital Psychiatry. 2010;32(1):66-72.

https://doi.org/10.1016/j.genhosppsych.2009.09.010

19. Mols F, Denollet J. Type D personality in the general population: a systematic review of health status, mechanisms of disease, and work-related problems. Health and Quality of Life
Outcomes. 2010;8:9.

https://doi.org/10.1186/1477-7525-8-9

20. Son HM. Verification for the validity and reliability of the Type D Scale-14. Journal of Korean Academy of Fundamentals of Nursing. 2008;15(3):312-20.

21. Higgins JP, Green S. Cochrane handbook for systematic reviews of interventions. England: John Wiley \& Sons, Ltd; 2008. p. 81-388.

22. Moher D, Liberati A, Tetzlaff J, Altman DG. Preferred reporting items for systematic reviews and meta-analyses: the PRISMA statement. BMJ. 2009;339:b2535. https://doi.org/10.1136/bmj.b2535

23. The Joanna Briggs Institute. The Joanna Briggs Institute critical appraisal tools for use in JBI systematic reviews [Internet]. Adelaide: Joanna Briggs Institute; 2017 [cited 2019 February 11]. Available from:

http://joannabriggs.org/research/critical-appraisal-tools.html

24. Sahoo S, Padhy SK, Padhee B, Singla N, Sarkar S. Role of personality in cardiovascular diseases: an issue that needs to be focused too! Indian Heart Journal. 2018;70(suppl 3):S471-7. https://doi.org/10.1016/j.ihj.2018.11.003

25. Hunt-Shanks T, Blanchard C, Reid R, Fortier M, Cappelli M. A psychometric evaluation of the Hospital Anxiety and Depression Scale in cardiac patients: addressing factor structure and gender invariance. British Journal of Health Psychology. 2010;15(1):97-114. https://doi.org/10.1348/135910709x432745

26. Lee SY, Choi SC, NA YH. A study on quality of life related to health. Journal of Neurogastroenterology and Motility. 2001;7 (1):6-17.

27. Thompson DR, Ski CF, Garside J, Astin F. A review of healthrelated quality of life patient-reported outcome measures in cardiovascular nursing. European Journal of Cardiovascular Nursing. 2016;15(2):114-25. https://doi.org/10.1177/1474515116637980

28. Denollet J, Conraads VM. Type D personality and vulnerability to adverse outcomes in heart disease. Cleveland Clinic Journal of Medicine. 2011;78(suppl 1):S13-9. https://doi.org/10.3949/ccjm.78.s1.02 


\section{Appendix 1. Studies Included in Systematic Review}

A1. Annagür BB, Demir K, Avci A, Uygur ÖF. Impact of a Type D personality on clinical and psychometric properties in a sample of Turkish patients with a first myocardial infarction. Journal of Psychiatric Practice. 2017;23(1):3-10. https://doi.org/10.1097/PRA.0000000000000201

A2. Bunevicius A, Staniute M, Brozaitiene J, Stropute D, Bunevicius R, Denollet J. Type D (distressed) personality and its assessment with the DS14 in Lithuanian patients with coronary artery disease. Journal of Health Psychology. 2013;18(9):124251. https://doi.org/10.1177/1359105312459098

A3. Cha KS, Im SM, Cho O-H. Mental health and quality of life by Type-D personality of the patients with coronary artery disease. The Journal of the Korean Content Association. 2013; 13(5):286-94. https://doi.org/10.5392/JKCA.2013.13.05.286

A4. Choi YO. A comparison of factors influencing health behavior compliance by Type D personality in the patients with coronary artery disease [master's thesis]. Gwangju: Chonnam National University; 2013.

A5. Condén E, Rosenblad A, Ekselius L, Åslund C. Prevalence of Type D personality and factorial and temporal stability of the DS14 after myocardial infarction in a Swedish population. Scandinavian Journal of Psychology. 2014;55(6):601-60. https://doi.org/10.1111/sjop.12162

A6. De Fazio P, Caroleo M, Rizza P, Cerminara G, De Serio D, Indolfi $\mathrm{C}$, et al. Specific personality traits and coping styles predict affective symptoms in early post acute coronary syndrome inpatients. The International Journal of Psychiatry in Medicine. 2012;44(2):119-32.

https://doi.org/10.2190/PM.44.2.c

A7. Denollet J. Personality and coronary heart disease: The TypeD Scale-16 (DS16). Annals of Behavioral Medicine. 1998;20 (3):209-15. https://doi.org/10.1007/BF02884962

A8. Doyle F, McGee HM, Conroy RM, Delaney M. What predicts depression in cardiac patients: sociodemographic factors, disease severity or theoretical vulnerabilities? Psychology \& Health. 2011;26(5):619-34. https://doi.org/10.1080/08870441003624398

A9. Kaur S, Zainal NZ, Low WY, Ramasamy R, Sidhu JS. Factor structure of hospital anxiety and depression scale in Malaysian patients with coronary artery disease. Asia Pacific Journal of Public Health. 2015;27(4):450-60. https://doi.org/10.1177/1010539514533719

A10. Kelpis TG, Anastasiadis K, Nimatoudis I, Kelpi MG, Hadjimiltiades S, Papakonstantinou C. Prevalence of "distressed" personality in patients with coronary artery disease and its correlation with morbidity after coronary surgery. Hellenic Journal of Cardiology. 2013;54(5):362-7.

A11. Lambertus F, Herrmann-Lingen C, Fritzsche K, Hamacher S,
Hellmich M, Jünger J, et al. Prevalence of mental disorders among depressed coronary patients with and without Type D personality. Results of the multi-center SPIRR-CAD trial. General Hospital Psychiatry. 2018;50:69-75. https://doi.org/10.1016/j.genhosppsych.2017.10.001

A12. Lee KH. Depression and health status in patient with Type D personality coronary artery disease [master's thesis]. Seoul: Korea University; 2015.

A13. Lee SJ, Koh S, Kim BO, Kim B, Kim C. Effect of Type D personality on short-term cardiac rehabilitation in patients with coronary artery disease. Annals of Rehabilitation Medicine. 2018;42(5):748-57. https://doi.org/10.5535/arm.2018.42.5.748

A14. Lim HE, Lee M-S, Ko Y-H, Park Y-M, Joe S-H, Kim Y-K, et al. Assessment of the Type D personality construct in the Korean population: a validation study of the Korean DS14. Journal of Korean Medical Science. 2011;26(1):116-23. https://doi.org/10.3346/jkms.2011.26.1.116

A15. Lin I-M, Wang S-Y, Chu I-H, Lu Y-H, Lee C-S, Lin T-H, et al. The association of Type $\mathrm{D}$ personality with heart rate variability and lipid profiles among patients with coronary artery disease. International Journal of Behavioral Medicine. 2017; 24(1):101-9. https://doi.org/10.1007/s12529-016-9571-x

A16. Middel B, El Baz N, Pedersen SS, van Dijk JP, Wynia K, Reijneveld SA. Decline in health-related quality of life 6 months after coronary artery bypass graft surgery: the influence of anxiety, depression, and personality traits. Journal of Cardiovascular Nursing. 2014;29(6):544-54. https://doi.org/10.1097/JCN.0b013e3182a102ae

A17. Molloy GJ, Perkins-Porras L, Strike PC, Steptoe A. Type-D personality and cortisol in survivors of acute coronary syndrome. Psychosomatic Medicine. 2008;70(8):863-8. https://doi.org/10.1097/psy.0b013e3181842e0c

A18. Molloy GJ, Randall G, Wikman A, Perkins-Porras L, MesserliBürgy N, Steptoe A. Type D personality, self-efficacy, and medication adherence following an acute coronary syndrome. Psychosomatic Medicine. 2012;74(1):100-6. https://doi.org/10.1097/PSY.0b013e31823a5b2f

A19. Mommersteeg PMC, Pot I, Aarnoudse W, Denollet J, Widdershoven JW. Type D personality and patient-perceived health in nonsignificant coronary artery disease: the Tweesteden Mild Stenosis (TWIST) study. Quality of Life Research. 2013;22(8):2041-50. https://doi.org/10.1007/s11136-012-0340-2

A20. Ossola P, De Panfilis C, Tonna M, Ardissino D, Marchesi C. DS14 is more likely to measure depression rather than a personality disposition in patients with acute coronary syndrome. Scandinavian Journal of Psychology. 2015;56(6):685-92. 
https://doi.org/10.1111/sjop.12244

A21. Park J-H, Tahk S-J, Bae S-H. Impact of Type D personality on health status and health behaviors in patients with coronary artery disease. Korean Journal of Health Promotion. 2010;10 (3):123-30.

A22. Pedersen SS, Denollet J, van Gestel YRBM, Serruys PW, van Domburg RT. Clustering of psychosocial risk factors enhances the risk of depressive symptoms 12-months post percutaneous coronary intervention. European Journal of Preventive Cardiology. 2008;15(2):203-9. https://doi.org/10.1097/HJR.0b013e3282f19d2f

A23. Pelle AJ, Erdman RAM, van Domburg RT, Spiering M, Kazemier M, Pedersen SS. Type D patients report poorer health status prior to and after cardiac rehabilitation compared to non-Type D patients. Annals of Behavioral Medicine. 2008;36(2):167-75. https://doi.org/10.1007/s12160-008-9057-4

A24. Smith ORF, Pedersen SS, van Domburg RT, Denollet J. Symptoms of fatigue and depression in ischemic heart disease are driven by personality characteristics rather than disease stage: a comparison of CAD and CHF patients. European Journal of Preventive Cardiology. 2008;15(5):583-8. https://doi.org/10.1097/HJR.0b013e3283083e17

A25. Son H-M. Quality of life and illness intrusiveness by Type-D personality in the patients with coronary artery disease. Journal of Korean Academy of Nursing. 2009;39(3):349-56. https://doi.org/10.4040/jkan.2009.39.3.349

A26. Spindler H, Pedersen SS, Serruys PW, Erdman RAM, van Domburg RT. Type-D personality predicts chronic anxiety following percutaneous coronary intervention in the drugeluting stent era. Journal of Affective Disorders. 2007;99(1-3): 173-9. https://doi.org/10.1016/j.jad.2006.09.009

A27. Svansdottir E, van den Broek KC, Karlsson HD, Gudnason T, Denollet J. Type D personality is associated with impaired psychological status and unhealthy lifestyle in Icelandic cardiac patients: a cross-sectional study. BMC Public Health. 2012;12:42. https://doi.org/10.1186/1471-2458-12-42

A28. van Gestel YRBM, Pedersen SS, van de Sande M, de Jaegere PPT, Serruys PW, Erdman RAM, et al. Type-D personality and depressive symptoms predict anxiety 12 months post-percutaneous coronary intervention. Journal of Affective Disorders. 2007;103(1-3):197-203.

https://doi.org/10.1016/j.jad.2007.01.030

A29. Vilchinsky N, Yaakov M, Sigawi L, Leibowitz M, Reges O, Levit $\mathrm{O}$, et al. Preliminary evidence for the construct and concurrent validity of the DS14 in Hebrew. International Journal of Behavioral Medicine. 2012;19(2):234-40. https://doi.org/10.1007/s12529-011-9147-8

A30. Vukovic O, Tosevski DL, Jasovic-Gasic M, Damjanovic A, Zebic M, Britvic D, et al. Type D personality in patients with coronary artery disease. Psychiatria Danubina. 2014;26(1):4651.

A31. Williams L, O'Connor RC, Grubb NR, O'Carroll RE. Type D personality and three-month psychosocial outcomes among patients post-myocardial infarction. Journal of Psychosomatic Research. 2012;72(6):422-6. https://doi.org/10.1016/j.jpsychores.2012.02.007 